\title{
A CRACK IN AN ANISOTROPIC ELASTIC SLAB*
}

\author{
BY \\ D. L. CLEMENTS \\ University of Adelaide
}

1. Introduction. The determination of the stress field round a crack in an infinite material has been the subject of a large number of studies during the last fifty years. The assumption that the material is infinite is, in most practical situations, justified by the fact that the crack is usually small compared with the dimensions of the material. Hence the effect of the boundary of the material on the stress field round the crack will be negligible provided the crack is sufficiently removed from the boundary. This assumption is acceptable for a large class of anisotropic materials and hence, for example, the solutions obtained by Stroh [1] and Clements [2,3] for cracks in infinite anisotropic materials will, in many cases, give relevant information about cracks in finite materials. However, if the material exhibits "strong anisotropy" or, more specifically, is virtually inextensible in a particular direction, then there may be a strong interaction between the boundary and the crack even though the two may be far removed from each other. In such cases the solution for the corresponding crack in an infinite material can give misleading results and it therefore seems desirable to obtain some exact solutions for crack problems in finite anisotropic materials. This is particularly the case in the light of recent interest in fiber-reinforced materials since such materials are often considered to be anisotropic in nature and to be almost inextensible in the fiber direction (see, for example, England and Rogers [4] and Everstine and Pipkin [5]). Thus, in the present paper, the problem of a crack in a strip of finite width and infinite length is considered. The problem of determining the stress field and crack energy is reduced to a set of three simultaneous Fredholm equations which may be solved numerically. Numerical values for the crack energy are obtained for some particular transversely isotropic materials and the results compared with those for the corresponding crack in an infinite material.

2. Statement of the problem and basic equations. Take Cartesian coordinates $x_{1}, x_{2}, x_{3}$ in a homogeneous anisotropic slab of elastic material occupying the region between the planes $x_{2}= \pm h$. In the plane $x_{2}=0$ there exists a crack in the region $\left|x_{1}\right|<a,-\infty<x_{3}<\infty$. The planes $x_{2}= \pm h$ are stress-free while the crack is opened by equal and opposite tractions on each side of the crack. It is required to find the stress distribution in the slab.

The necessary basic equations are derived in Clements [2]. The expressions for the displacements $u_{k}$ and stresses $\sigma_{i j}$ are

$$
u_{k}=2 \Re \sum_{\alpha} A_{k \alpha} \chi_{\alpha}\left(z_{\alpha}\right), \quad \sigma_{i j}=2 \Re \sum_{\alpha} L_{i j \alpha} \chi_{\alpha}{ }^{\prime}\left(z_{\alpha}\right),
$$

* Received November 11, 1975. 
where $R$ denotes the real part of a complex number, the $\chi_{\alpha}\left(z_{\alpha}\right)(\alpha=1,2,3)$ are analytic functions of the complex variable $z_{\alpha}=x_{1}+\tau_{\alpha} x_{2}$ and primes denote derivatives with respect to the argument in question. Also, in (2.1) the $A_{k \alpha}$ satisfy the equations

$$
\left(c_{i 1 k 1}+\tau_{\alpha} c_{i 1 k 2}+\tau_{\alpha} c_{i 2 k 1}+\tau_{\alpha}{ }^{2} c_{i 2 k 2}\right) A_{k \alpha}=0
$$

where the convention of summing over a repeated Latin suffix is used and the $\tau_{\alpha}$ are the roots with positive imaginary part of the sextic equation

$$
\left|c_{i 1 k 1}+\tau c_{i 1 k 2}+\tau c_{i 2 k 1}+\tau^{2} c_{i 2 k 2}\right|=0,
$$

where the $c_{i j k l}$ are the elastic constants. Finally the $L_{i j \alpha}$ occurring in (2.2) are related to the $A_{k \alpha}$ by the equation

$$
L_{i j \alpha}=\left(c_{i j k 1}+\tau_{\alpha} c_{i j k 2}\right) A_{k \alpha} .
$$

3. Solution of the problem. In order to satisfy the boundary conditions it is useful to write the displacement and stress as the sum of three separate displacement and stress fields. We write

$$
u_{k}=u_{k}{ }^{(1)}+u_{k}{ }^{(2)}+u_{k}{ }^{(3)}, \quad \sigma_{i j}=\sigma_{i j}{ }^{(1)}+\sigma_{i j}{ }^{(2)}+\sigma_{i j}{ }^{(3)} .
$$

Suitable forms for $u_{k}^{(1)}$ and $u_{k}^{(2)}$ are obtained by referring to the work of Tauchert [6] and Tauchert and Aköz [7]. For $u_{k}{ }^{(1)}$ and $\sigma_{i i}{ }^{(1)}$ we substitute

$$
\chi_{\alpha}\left(z_{\alpha}\right)=\frac{1}{2 \pi} \int_{0}^{\infty} E_{\alpha}(p) \exp \left(i p z_{\alpha}\right) d p
$$

in (2.1) and (2.2) to obtain

$$
\begin{aligned}
u_{k}{ }^{(1)} & =\frac{1}{\pi} \Re \int_{0}^{\infty} \sum_{\alpha} A_{k \alpha} E_{\alpha}(p) \exp \left(i p z_{\alpha}\right) d p, \\
\sigma_{i j}{ }^{(1)} & =\frac{1}{\pi} \propto \int_{0}^{\infty} \sum_{\alpha} L_{i i_{\alpha}} E_{\alpha}(p) i p \exp \left(i p z_{\alpha}\right) d p .
\end{aligned}
$$

Similarly, for $u_{k}{ }^{(2)}$ and $\sigma_{i i}{ }^{(2)}$ we substitute

$$
\chi_{\alpha}\left(z_{\alpha}\right)=\frac{1}{2 \pi} \int_{0}^{\infty} F_{\alpha}(p) \exp \left(-i p z_{\alpha}\right) d p
$$

in (2.1) and (2.2) to obtain

$$
\begin{aligned}
u_{k}{ }^{(2)} & =\frac{1}{\pi} \Re \int_{0}^{\infty} \sum_{\alpha} A_{k \alpha} F_{\alpha}(p) \exp \left(-i p z_{\alpha}\right) d p, \\
\sigma_{i j}{ }^{(2)} & =-\frac{1}{\pi} \Re \int_{0}^{\infty} \sum_{\alpha} L_{i j \alpha} F_{\alpha}(p) i p \exp \left(-i p z_{\alpha}\right) d p .
\end{aligned}
$$

For $u_{k}{ }^{(3)}$ and $\sigma_{i j}{ }^{(3)}$ we consider the regions $0<x_{2}<h$ and $-h<x_{2}<0$ separately. For $0<x_{2}<h$ the expressions for $u_{k}{ }^{(3)}$ and $\sigma_{i j}{ }^{(3)}$ are

$$
\begin{aligned}
u_{k}{ }^{(3)} & =\frac{1}{\pi} \propto \int_{0}^{\infty} \sum_{\alpha} A_{k \alpha} G_{\alpha}{ }^{+}(p) \exp \left(i p z_{\alpha}\right) d p, \\
\sigma_{i j}{ }^{(3)} & =\frac{1}{\pi} \propto \int_{0}^{\infty} \sum_{\alpha} L_{i j \alpha} G_{\alpha}{ }^{+}(p) i p \exp \left(i p z_{\alpha}\right) d p .
\end{aligned}
$$


In $-h<x_{2}<0$ the corresponding expressions are

$$
\begin{aligned}
u_{k}^{(3)} & =\frac{1}{\pi} \Re \int_{0}^{\infty} \sum_{\alpha} A_{k \alpha} G_{\alpha}{ }^{-}(p) \exp \left(-i p z_{\alpha}\right) d p, \\
\sigma_{i i}{ }^{(3)} & =-\frac{1}{\pi} \propto \int_{0}^{\infty} \sum_{\alpha} L_{i j \alpha} G_{\alpha}{ }^{-}(p) i p \exp \left(-i p z_{\alpha}\right) d p .
\end{aligned}
$$

The stresses $\sigma_{i 2}$ must be continuous across $x_{2}=0$ and hence, from (3.2), (3.5), (3.8), (3.10) and (3.12), it follows that

$$
\sum_{\alpha} L_{i 2 \alpha} G_{\alpha}{ }^{+}(p)=\sum_{\alpha} \bar{L}_{i 2 \alpha} \bar{G}_{\alpha}^{-}(p) .
$$

Denoting these expressions by $\psi_{i}(p)$, we obtain

$$
\begin{aligned}
& G_{\alpha}{ }^{+}(p)=M_{\alpha i} \psi_{i}(p), \\
& G_{\alpha}{ }^{-}(p)=M_{\alpha_{i}} \bar{\psi}_{i}(p),
\end{aligned}
$$

where

$$
\sum_{\alpha} L_{i 2 \alpha} M_{\alpha i}=\delta_{i i} .
$$

From (3.9), (3.11), (3.14) and (3.15) it is apparent that the difference in displacement on either side of the plane $x_{2}=0$ is

$$
\Delta u_{k}=\frac{1}{\pi} \mathfrak{R}\left(B_{k i}-\bar{B}_{k i}\right) \int_{0}^{\infty} \psi_{i}(p) \exp \left(i p x_{1}\right) d p,
$$

where

$$
B_{k i}=\sum_{\alpha} A_{k \alpha} M_{\alpha i}
$$

The difference in displacement $\Delta u_{k}$ outside the crack must be zero and hence, from (3.17),

$$
\mathcal{R}\left(B_{k i}-\bar{B}_{k j}\right) \int_{0}^{\infty} \psi_{i}(p) \exp \left(i p x_{1}\right) d p=0 \text { for }\left|x_{1}\right|>a .
$$

From (3.5), (3.8), (3.10), (3.14) and (3.16) it follows that the stress boundary condition on the crack faces will be satisfied if

$$
\begin{aligned}
& \frac{1}{\pi} \Re \int_{0}^{\infty}\left[\psi_{i}(p)+\sum_{\alpha}\left\{L_{i 2 \alpha} E_{\alpha}(p)+\bar{L}_{i 2 \alpha} \bar{F}_{\alpha}(p)\right\}\right] i p \exp \left(i p x_{1}\right) d p=-P_{i}\left(x_{1}\right) \\
& \text { for }\left|x_{1}\right|<a,
\end{aligned}
$$

where the $P_{j}\left(x_{1}\right)$ are the given surface tractions.

The final boundary condition concerns the requirement that the tractions be zero on $x_{2}= \pm h$. From (3.2), (3.5), (3.8), (3.10) and (3.12) it follows that this condition will be satisfied if

$$
\begin{aligned}
& \sum_{\alpha}\left[L_{i 2 \alpha}\left\{E_{\alpha}(p)+G_{\alpha}{ }^{+}(p)\right\} \exp \left(i p \tau_{\alpha} h\right)+\bar{L}_{i 2 \alpha} \bar{F}_{\alpha}(p) \exp \left(i p \bar{\tau}_{\alpha} h\right)\right]=0, \\
& \sum_{\alpha}\left[L_{i 2 \alpha} E_{\alpha}(p) \exp \left(-i p \tau_{\alpha} h\right)+\bar{L}_{i 2 \alpha}\left\{\bar{F}_{\alpha}(p)+\bar{G}_{\alpha}{ }^{-}(p)\right\} \exp \left(-i p \bar{\tau}_{\alpha} h\right)\right]=0
\end{aligned}
$$


If we require the applied tractions $P_{j}\left(x_{1}\right)$ to be even functions of $x_{1}$ then it is sufficient to put

$$
\psi_{i}(p)=i \int_{0}^{a} r_{i}(t) J_{0}(p t) d t
$$

where the $r_{i}(\mathrm{t})(j=1,2,3)$ are real functions to be determined and $J_{0}$ is the Bessel function of order zero. With this choice of the $\psi_{j}(p)$ the condition (3.19) is automatically satisfied. Furthermore, addition of the conjugate of (3.22) to (3.21) and use of (3.14), (3.15) and (3.23) yield

$$
\sum_{\alpha}\left\{L_{i 2 \alpha}\left[E_{\alpha}(p)+F_{\alpha}(p)\right] \exp \left(i p \tau_{\alpha} h\right)+\bar{L}_{i 2 \alpha}\left[\bar{E}_{\alpha}(p)+\bar{F}_{\alpha}(p)\right] \exp \left(i p \bar{\tau}_{\alpha} h\right)\right]=0
$$

It follows that

$$
E_{\alpha}(p)=-F_{\alpha}(p)
$$

and hence $(3.20)$ reduces to

$$
\begin{array}{r}
\int_{0}^{\infty} \cos \left(p x_{1}\right) p d p \int_{0}^{a} r_{j}(t) J_{0}(p t) d t+\int_{0}^{\infty} T_{i k}(p) \cos \left(p x_{1}\right) p d p \int_{0}^{a} r_{k}(t) J_{0}(p t) d t \\
=\pi P_{i}\left(x_{1}\right) \text { for }\left|x_{1}\right|<a
\end{array}
$$

where $T_{i k}(p)$ is the real matrix defined by

$$
T_{i k}(p)=\sum_{\alpha} L_{j 2 \alpha} Q_{\alpha k}(p)+\sum_{\alpha} \bar{L}_{i 2 \alpha} \bar{Q}_{\alpha k}(p) .
$$

with $Q_{\alpha k}$ given by

$$
E_{\alpha}(p)=Q_{\alpha k}(p) \psi_{k}(p)
$$

Numerical values of the elements of $Q_{\alpha k}$ for particular values of $p$ may be obtained by employing (3.14), (3.15), (3.21) and (3.22).

Now, by interchanging the order of integration in the first integral on the left-hand side of (3.26), it follows that

$$
\begin{aligned}
\frac{d}{d x_{1}} \int_{0}^{x_{1}} \frac{r_{i}(t) d t}{\left(x_{1}{ }^{2}-t^{2}\right)^{1 / 2}}+\int_{0}^{\infty} T_{i k}(p) \cos \left(p x_{1}\right) p d p \int_{0}^{a} r_{k}(t) J_{0}(p t) d t=\pi P_{i}\left(x_{1}\right) \\
\text { for } 0<x_{1}<a .
\end{aligned}
$$

Solving this Abel's equation for $r_{j}(t)$ and interchanging the order of integration in the resulting triple integral, we obtain

$$
r_{i}(t)+t \int_{0}^{a} K_{j k}(s, t) r_{k}(s) d s=2 t \int_{0}^{t} \frac{P_{i}(u) d u}{\left(t^{2}-u^{2}\right)^{1 / 2}} \quad \text { for } \quad 0<t<a,
$$

where

$$
K_{j k}(s, t)=\int_{0}^{\infty} T_{i k}(p) J_{0}(p s) J_{0}(p t) p d p
$$

Eqs. (3.30) constitute three simultaneous Fredholm integral equations for the $r_{j}(t)$, $j=1,2,3$. 
4. The crack energy. The energy of the crack is given by the integral

$$
U=\frac{1}{2} \int_{-a}^{a} P_{k}\left(x_{1}\right) \Delta u_{k} d x_{1},
$$

where $\Delta u_{k}$ is given by (3.17). Using (3.23) in (3.17) and changing the order of integration, we obtain

$$
\Delta u_{k}=\frac{1}{\pi} i\left(B_{k j}-\bar{B}_{k j}\right) \int_{0}^{a} \frac{r_{j}(t) d t}{\left(t^{2}-x_{1}^{2}\right)^{1 / 2}} \text { for } 0<x_{1}<a .
$$

Hence (4.1) yields

$$
U=\frac{i}{2 \pi}\left(B_{k i}-\bar{B}_{k i}\right) \int_{0}^{a} r_{i}(t) d t \int_{-t}^{t} \frac{P_{k}\left(x_{1}\right) d x_{1}}{\left(t^{2}-x_{1}^{2}\right)^{1 / 2}}
$$

In the case of constant applied tractions $P_{k}\left(x_{1}\right)=P_{k}(4.3)$ reduces to

$$
U=\frac{i}{2}\left(B_{k i}-\bar{B}_{k i}\right) P_{k} \int_{0}^{a} r_{i}(t) d t
$$

5. Numerical results. If we consider the case of constant applied tractions $P_{i}$ and put $t=a t^{\prime}, s=a s^{\prime}$ and $p=p^{\prime} / a$ then (3.30) may be written in the form

$$
r_{i}\left(a t^{\prime}\right)+t^{\prime} \int_{0}^{1} K_{j k}^{\prime}\left(s^{\prime}, t^{\prime}\right) r_{k}\left(a s^{\prime}\right) d s^{\prime}=a \pi t^{\prime} P_{i} \text { for } 0<t^{\prime}<1,
$$

where

$$
K_{i k}^{\prime}\left(s^{\prime}, t^{\prime}\right)=\int_{0}^{\infty} T_{j k}\left(p^{\prime} / a\right) J_{0}\left(p^{\prime} s^{\prime}\right) J_{0}\left(p^{\prime} t^{\prime}\right) p^{\prime} d p^{\prime}
$$

Also, substituting $t=a t^{\prime}$ in (4.4), we obtain

$$
U=\frac{i}{2}\left(B_{k i}-\bar{B}_{k i}\right) P_{k} a \int_{0}^{1} r_{i}\left(a t^{\prime}\right) d t^{\prime}
$$

In the particular case when the only applied traction is normal to the crack face, $P_{1}=$ $P_{3}=0$ and (5.1) and (5.3) reduce to

$$
\begin{gathered}
{\left[a^{-1} P_{2}^{-1} r_{j}\left(a t^{\prime}\right)\right]+t^{\prime} \int_{0}^{1} K_{i k}^{\prime}\left(s^{\prime}, t^{\prime}\right)\left[a^{-1} P_{2}^{-1} r_{k}\left(a s^{\prime}\right)\right] d s^{\prime}=\pi t^{\prime} \delta_{i 2}} \\
U^{\prime}=\frac{i}{2}\left(B_{2 i}-\bar{B}_{2 i}\right) P_{2}{ }^{2} a^{2} \int_{0}^{1}\left[a^{-1} P_{2}^{-1} r_{j}\left(a t^{\prime}\right)\right] d t^{\prime}
\end{gathered}
$$

In order to obtain some idea of the behavior of (5.5) in particular cases we consider an anisotropic material which is transversely isotropic.

The elastic behavior of transversely isotropic materials may be characterized by the five elastic constants $A, N, F, C$ and $L$. We may conveniently use two angles $\alpha$ and $\theta$ to describe the general orientation of the Cartesian axes within the material as follows. Suppose that initially (that is, when $\alpha=\theta=0$ ) the $x_{3}$-axis is normal to the transverse plane so that the $x_{1}$-axis and $x_{2}$-axis lie in the transverse plane. The $x_{2}$-axis is kept fixed and the $x_{1}$-axis and $x_{3}$-axis are rotated through an angle $\alpha$. The $x_{1}$-axis is then kept 
fixed in its new position at angle $\alpha$ to the transverse plane and the $x_{2}$-axis and $x_{3}$-axis are rotated through an angle $\theta$. Both of these rotations are in the positive direction. The $c_{i j k l}$ are then given by the equations

$$
c_{i j k l}=a_{i p} a_{i q} a_{k r} a_{l s} c_{p a r}{ }^{\prime}
$$

where

$$
\begin{gathered}
{\left[a_{i j}\right]=\left[\begin{array}{ccc}
\cos \alpha & 0 & -\sin \alpha \\
\sin \theta \sin \alpha & \cos \theta & \sin \theta \cos \alpha \\
\cos \theta \sin \alpha & -\sin \theta & \cos \theta \cos \alpha
\end{array}\right],} \\
c_{1111^{\prime}}=c_{2222}{ }^{\prime}=A, \quad c_{1122}{ }^{\prime}=N, \quad c_{1133}{ }^{\prime}=c_{2233}{ }^{\prime}=F^{\prime}, \\
c_{1313}{ }^{\prime}=c_{2332}{ }^{\prime}=L, \quad c_{1212}{ }^{\prime}=\frac{i}{2}(A-N), \quad c_{3333}{ }^{\prime} \quad C,
\end{gathered}
$$

and all other $c_{i j k l}{ }^{\prime}$ are zero.

We consider a material for which the elastic constants are $A=5.96, N=2.57$, $F=2.14, C=6.14$ and $L=1.64$. If each of these constants is multiplied by $10^{11}$ then the units for the constants are dynes $/ \mathrm{cm}^{2}$. These are the constants for a crystal of magnesium, although they are used here merely for illustrative purposes. By employing eqs. (4.3) and (4.4), the variation of the ratio $U / U_{0}$ (where $U_{0}$ denotes the energy of the corresponding crack in an infinite material) was calculated for $\alpha=0, \theta=\pi / 2$ and various values of $h / a$. The results are shown in Table 1 and indicate that, for the particular material under consideration, the difference between the energies of the crack in the strip and the crack in an infinite material is small provided the strip width is more than five times the crack length. If the strip width is less than three times the crack length then the difference in energies is appreciable. Similar results to those given in Table 1 were obtained for the angles $\alpha=\pi / 6, \theta=\pi / 4$ and $\alpha=\pi / 3, \theta=\pi / 4$ and hence

T.ABL: 1. Variation of crack energy with strip width for $\alpha=0$ and $\theta=\pi / 2$.

$\begin{array}{lccccccc}h / a & 1 & 2 & 3 & 4 & 5 & 10 & 20 \\ U / U_{0} & 2.19 & 1.31 & 1.14 & 1.08 & 1.05 & 1.01 & 1.00\end{array}$

it is perhaps reasonable to assume that this pattern would be repeated for any values of $\alpha$ and $\theta$.

Table 2. Variation of crack energy with $C$ for $\alpha=0, \theta=\pi / 2$ and $h / a=5$.

$\begin{array}{ccccccccccc}C & 10 & 20 & 30 & 40 & 50 & 60 & 70 & 80 & 90 & 100 \\ U / U_{0} & 1.07 & 1.13 & 1.18 & 1.23 & 1.27 & 1.31 & 1.34 & 1.38 & 1.41 & 1.45\end{array}$

TABLE 3. Variation of crack energy with $C$ for $\alpha=\pi / 6, \theta=\pi / 4$ and $h / a=5$.

$\begin{array}{ccccccccccr}C & 10 & 20 & 30 & 40 & 50 & 60 & 70 & 80 & 90 & 100 \\ U / U_{0} & 1.04 & 1.04 & 1.05 & 1.05 & 1.05 & 1.05 & 1.05 & 1.05 & 1.05 & 1.05\end{array}$

Taise 4. Variation of crack energy with $C$ for $\alpha=\pi / 3, \theta=\pi / 4$ and $h / a=20$.

$\begin{array}{ccccccccccc}C & 10 & 20 & 30 & 40 & 50 & 60 & 70 & 80 & 90 & 100 \\ U / U_{0} & 1.05 & 1.05 & 1.06 & 1.06 & 1.06 & 1.07 & 1.07 & 1.07 & 1.08 & 1.08\end{array}$


We now consider materials with the same values of $A, N, L$ and $F$ as for magnesium but with various values of $C$. As $C$ increases the extensibility of the material in a direction normal to the transverse plane decreases. As $C$ becomes large, such a material will be a reasonable model of a fiber-reinforced material with almost inextensible straight fibers in an elastic matrix, the direction of the fibers being normal to the transverse planes (see, for example, Clements [8]). The variation of $U / U_{0}$ for various values of $\alpha, \theta$ and $C$ is shown in Tables 2,3 and 4 . It is interesting to note that a substantial increase in $C$ only causes a marked change in $U / U_{0}$ in the case when $\alpha=0$ and $\theta=\pi / 2$. For these values of $\alpha$ and $\theta$ the crack lies in a transverse plane and the "fiber direction" is normal to the plane of the crack. If the "fiber direction" is at a substantial angle to the normal to the plane of the crack then, on the basis of the results in Tables 3 and 4, it is reasonable to conclude that an increase in inextensibility in the "fiber direction" has negligible effect on the crack energy.

In conclusion, the results show that for a material which is not "strongly anisotropic" it is reasonable to use the energy for a crack in an infinite material as an approximation for the energy of a crack in a strip provided the strip width is greater than roughly twenty times the crack length. If, however, the material is "strongly anisotropic" or, more particularly, is almost inextensible in a particular direction, then some care is needed in approximating a crack problem for a strip by a similar problem for an infinite material.

\section{REFERENCES}

[1] A. N. Stroh, Dislocations and cracks in anisotropic elasticity, Philos. Mag. (8) 3, 625-646 (1958)

[2] D. L. Clements, $A$ crack between dissimilar anisotropic media, Int. J. Engng. Sc. 9, 257-265 (1971)

[3] D. L. Clements, A crack between isotropic and anisotropic media, Quart. Appl. Math. 29, 303-310 (1971)

[4] A. H. England and T. G. Rogers, Plane crack problems for ideal fibre-reinforced materials, Quart. J. Mech. Appl. Math. 26, 303-320 (1973)

[5] G. C. Everstine and A. C. Pipkin, Boundary layers in fiber-reinforced materials, J. Appl. Mech. Ser. E40, 518-522 (1973)

[6] T. R. Tauchert, Stress in an anisotropic eiastic slab due to distributed surface loads and displacements, in Proc. 14th Midwestern Mechs. Conf., 89-101 (1975)

[7] T. R. Tauchert and A. Y. Aköz, Stationary temperature and stress fields in an anisotropic elastic slab, J. Appl. Mech. Ser. E42, 647-650 (1975)

[8] D. L. Clements, $A$ property of contact problems for anisotropic and fiber-reinforced half-spaces, Utilitas Mathematica 2, 33-46 (1972) 